Original Research Article

\title{
Antimicrobial susceptibility pattern of urine culture isolates in a tertiary care hospital of Jharkhand, India
}

\author{
Akhilesh Kumar, Rajiv Kumar*, Manju Gari, Umashanker P. Keshri, \\ Sumit K. Mahato, Kumari Ranjeeta
}

Department of Pharmacology, Rajendra Institute of Medical Sciences (RIMS), Ranchi, Jharkhand, India

Received: 29 April 2017 Accepted: 24 May 2017

*Correspondence to:

Dr. Rajiv Kumar,

Email: kolgong146@gmail.com

Copyright: (C) the author(s), publisher and licensee Medip Academy. This is an openaccess article distributed under the terms of the Creative Commons Attribution NonCommercial License, which permits unrestricted noncommercial use, distribution, and reproduction in any medium, provided the original work is properly cited.

\begin{abstract}
Background: Urinary tract infection (UTI) is one of the common infections encountered by the clinicians. Though a good number of antimicrobial agents are available, still UTIs have become difficult to treat due to development of resistance by the uropathogens. So, regional data regarding the common uropathogens and their sensitivity pattern is required to guide the clinicians to start empirical therapy while managing UTIs. The purpose of the study was to identify different species of microorganisms, along with their antimicrobial susceptibility pattern, causing urinary tract infection in outpatient and indoor patients at RIMS, Ranchi, Jharkhand.

Methods: Observational study was conducted using urine culture and sensitivity reports collected retrospectively from records maintained in the department of Microbiology over a period from July 2016 to Feb 2017 in tertiary care hospital.

Results: UTI was more common in females $(57.74 \%)$ than in males $(42.26 \%)$. Among the uropathogens isolated Escherichia coli $(37.41 \%)$ was found to be the predominant organism followed by Klebsiella species (32.79\%), Pseudomonas species (25.86\%), and gram-positive bacteria Staphylococcus aureus accounted $(3.92 \%)$ of total cases. The most common isolates were $E$. coli showed high sensitivity to amikacin (79.24\%), followed by levofloxacin $(77.21 \%)$ and gentamycin $(62.26 \%)$. It was found to be resistant to norfloxacin (86\%), nalidixic acid $(86.76 \%)$ and cefotaxime $(69.88 \%)$.

Conclusions: Though various microorganisms are responsible for UTI. Escherichia coli species is the most common organism. Antimicrobial resistance has already emerged against many antibiotics, making empirical treatment of these infections challenging.
\end{abstract}

Keywords: Antimicrobial susceptibility pattern, Bacterial isolates, Urine culture, Urinary tract infection

\section{INTRODUCTION}

UTI is the most common bacterial infection accounting for $25 \%$ of all infections. It is one of the most important causes of morbidity and also the second most common cause of hospital visit. ${ }^{1}$ UTIs are defined by the presence of a growth of more han $10^{5}$ colony forming units (CFU) of bacteria per $\mathrm{ml}$ of urine for asymptomatic individual and much lower for symptomatic individual $\left(\sim 10^{3}\right.$ $\mathrm{CFU} / \mathrm{ml}){ }^{2}$ In urine sample obtained by supra pubic aspiration or catheterization and in samples from a patient with an indwelling catheter, colony count of $10^{2}-10^{4} / \mathrm{ml}$ generally indicates infection. ${ }^{3}$ Indwelling urinary catheters pose a risk for many infective complications such as perinephric, vesical, and urethral abscesses as well as epididymitis, prostatitis and orchitis. The overall incidence of these complications is unknown, although 20 to 30 percent of patients with asymptomatic catheterinduced UTIs may develop local or systemic symptoms. ${ }^{4}$ Women are more prone to UTIs, probably because they have shorter urethras which therefore pass bacteria into the urinary tract more easily. ${ }^{5}$ Every woman has a $60 \%$ lifetime risk of developing bacterial cystitis, which develops mostly before the age of $24 .{ }^{6}$ In contrast, men 
have a lifetime risk of only $13 \% .^{6}$ In children approximately $5 \%$ of girls and $1 \%$ of boys have a UTI by 11 years of age. ${ }^{7}$ It is also the most common cause of nosocomial infections in adults. The vast majority of uncomplicated UTIs are caused by the gram-negative bacillus i.e. Escherichia coli, along with other pathogens including Enterococci, Staphylococcus saprophyticus, Klebsiella spp. and Proteus mirabilis. In patients with suspected UTI, antibiotic treatment is usually started empirically, before urine culture results are available. To ensure appropriate treatment, knowledge of the organisms that cause UTI and their antibiotic susceptibility is mandatory. Local condition can modify these data; they need to be constantly re-evaluated to achieve a maximal clinical response before the antibiotic susceptibility of the isolate is known. The aim of the present study was to assess the changing susceptibility pattern of urinary pathogens to antimicrobial agents in UTIs.

\section{METHODS}

A total of 1723 urine culture sensitivity reports were analyzed of patients who were suspected to be having urinary tract infection, from July 2016 to Feb 2017 with prior Clean-catch midstream urine specimens from patients diagnosed clinically to be having UTI on the basis of symptoms (fever, dysuria and increased frequency of urination) were inoculated on Blood Agar and McConkey Agar plates, which were incubated aerobically at $37^{\circ} \mathrm{C}$ overnight. Plates showing growth suggestive of significant bacteruria, with colony counts exceeding $10^{5} \mathrm{cfu} / \mathrm{ml}$ were subjected to standard biochemical tests for identification and antimicrobial sensitivity testing by Kirby- Bauer disc diffusion method. Interpretation as 'Sensitive' or 'Resistant' was done on the basis of the diameters of zones of inhibition of bacterial growth as recommended by the disc manufacturer. Antibiotics against which sensitivity was tested in the present study included amoxycillin, amoxiclav, ciprofloxacin, norfloxacin, levofloxacin, cotrimoxazole, gentamicin, amikacin, nitrofurantoin, imipenem, meropenem and cefotaxime.

\section{RESULTS}

A total of 1723 sample were studied Retrospectively out of which 433 came out positive for culture. Among these $433,183(42.26 \%)$ samples belonged to male and 250 $(57.74 \%)$ belonged to female patient as shown in Figure 1. The most common isolates in this study have been the Gram-negative bacilli which accounts for $76.78 \%$ of the total positive isolates. In the gram-negative bacilli, the predominant isolate was the Enterobacter spp. $(\mathrm{n}=162$, $37.41 \%)$ followed by Klebsiella spp. $(\mathrm{n}=142,32.79 \%)$, and Pseudomonas $(\mathrm{n}=112,25.86 \%)$ among the major isolates. In the gram-positive bacteria, the main organism identified was Staphylococcus aureus $(\mathrm{n}=17,3.92 \%)$. Table 1 shows the detailed frequency of all the isolates identified.

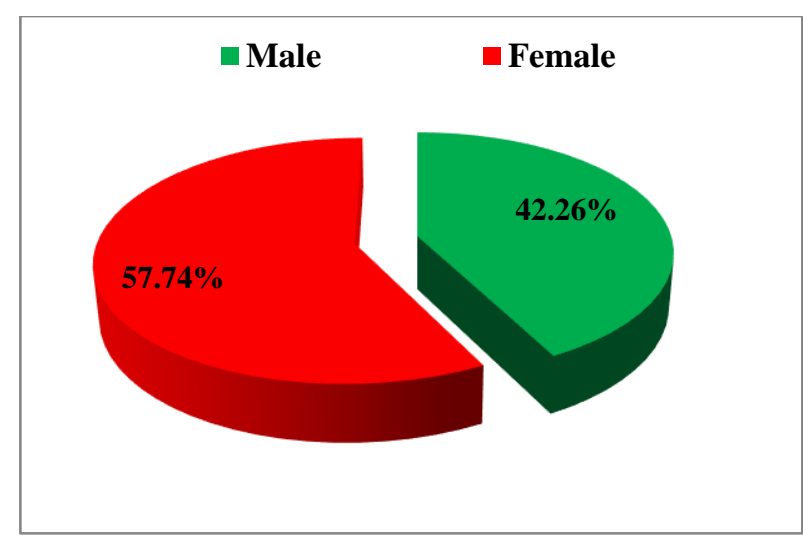

Figure 1: Sex wise distribution of UTI in percentage.

Table 1: Species isolated, their number and percentage from urine culture.

\begin{tabular}{|lll|}
\hline Species isolated & Number & Percentage \\
\hline E. coli & 162 & $37.41 \%$ \\
\hline Klebsiella & 142 & $32.79 \%$ \\
\hline Pseudomonas & 112 & $25.86 \%$ \\
\hline Staphylococcus & 17 & $3.92 \%$ \\
\hline
\end{tabular}

The most common isolates were E. coli $(37.41 \%)$ showed high sensitivity to imipenem $(90.80 \%)$, followed by meropenem $(85.40 \%)$, amikacin $(79.24 \%)$ levofloxacin (77.21\%), gentamycin (62.26\%) and ampicillin (59.92\%). It was found to be resistant to cotrimoxazole (96.14\%), norfloxacin $(88 \%)$, nalidixic acid $(86.76 \%)$, cefotaxime $(69.88 \%)$, ciprofloxacin $(55.07 \%)$ and nitrofurantoin $(40.65 \%)$ as shown in Table 2.

Table 2: Susceptibility pattern of $E$. coli.

\begin{tabular}{|lll|l|}
\hline E. coli & H.S & I.S & R \\
\hline Imipenem & $90.80 \%$ & $8.31 \%$ & $1.76 \%$ \\
\hline Meropenem & $85.40 \%$ & $12.63 \%$ & $2.67 \%$ \\
\hline Amikacin & $79.24 \%$ & $\%$ & $20.75 \%$ \\
\hline Levofloxacin & $77.21 \%$ & $7.61 \%$ & $15.18 \%$ \\
\hline Gentamycin & $62.26 \%$ & $\%$ & $37.73 \%$ \\
\hline Ampicillin & $59.92 \%$ & $17.07 \%$ & $23 \%$ \\
\hline Nitrofurantoin & $51.22 \%$ & $8.13 \%$ & $40.65 \%$ \\
\hline Ciprofloxacin & $44.92 \%$ & $\%$ & $55.07 \%$ \\
\hline Cefotaxime & $17.22 \%$ & $27.89 \%$ & $69.88 \%$ \\
\hline Nalidixic acid & $\%$ & $13.23 \%$ & $86.76 \%$ \\
\hline Norfloxacin & $\%$ & $12 \%$ & $88 \%$ \\
\hline Cotrimoxazole & & $3.80 \%$ & $96.14 \%$ \\
\hline
\end{tabular}

The second most common isolates Klebsiella showed high sensitivity to cefoperazone with sulbactum (100\%), imipenem (92\%), aminoglycosides like gentamicin (69.64\%) and amikacin (59.79\%). It showed high resistance to amoxicillin (100\%), nalidixic acid $(72.41 \%)$, norfloxacin $(55.07 \%)$, as shown in Table 3. 
Table 3: Susceptibility pattern of Klebsiella.

\begin{tabular}{|llll|}
\hline Klebsiella & H.S & I.S & R \\
\hline $\begin{array}{l}\text { Cefoperazone } \\
\text { with sulbactum }\end{array}$ & $100 \%$ & $\%$ & $\%$ \\
\hline imipenem & $92 \%$ & $7.79 \%$ & \\
\hline gentamycin & $69.64 \%$ & $30.35 \%$ & $\%$ \\
\hline amikacin & $59.79 \%$ & $39.17 \%$ & $\%$ \\
\hline levofloxacin & $40 \%$ & $38.09 \%$ & $18.09 \%$ \\
\hline ciprofloxacin & $32.50 \%$ & $32.50 \%$ & $35 \%$ \\
\hline Nitrofurantoin & $25.80 \%$ & $49.46 \%$ & $24.73 \%$ \\
\hline norfloxacin & $11.59 \%$ & $33.33 \%$ & $55.07 \%$ \\
\hline Nalidixic acid & $\%$ & $27.58 \%$ & $72.41 \%$ \\
\hline Amoxicillin & $\%$ & $\%$ & $100 \%$ \\
\hline
\end{tabular}

H.S =high sensitivity, I.S =Intermediate sensitivity, R=Resistant

Pseudomonas which is the $3^{\text {rd }}$ most predominant isolate gave high sensitivity to imipenem $(87 \%)$ ceftriaxone with sulbactum (75\%) and amikacin 55.47\%. Among the tested antibiotics it showed high resistance to amoxicillin $(87.50 \%)$, cefotaxime $(81.37 \%)$, nalidixic acid $(70.11 \%)$, ampicillin $(55 \%)$, norfloxacin $(52.83 \%)$, ciprofloxacin $(35.46 \%)$ respectively.as shown in Table 4.

\section{Table 4: Susceptibility pattern of Pseudomonas aeruginosa.}

\begin{tabular}{|c|c|c|c|}
\hline \multicolumn{4}{|c|}{ Pseudomonas aeruginosa } \\
\hline & H.S & I.S & $\mathbf{R}$ \\
\hline Imipenem & $87 \%$ & $13 \%$ & $\%$ \\
\hline $\begin{array}{l}\text { Ceftriaxone with } \\
\text { sulbactum }\end{array}$ & $75 \%$ & $25 \%$ & $\%$ \\
\hline Amikacin & $55.47 \%$ & $44.52 \%$ & $\%$ \\
\hline $\begin{array}{l}\text { Cefoperazone with } \\
\text { sulbactum }\end{array}$ & $50 \%$ & $50 \%$ & $\%$ \\
\hline Lomefloxacin & $50 \%$ & $50 \%$ & $\%$ \\
\hline Ofloxacin & $50 \%$ & $50 \%$ & $\%$ \\
\hline $\begin{array}{l}\text { Piperacillin with } \\
\text { tazobactum }\end{array}$ & $45.83 \%$ & $54.16 \%$ & $\%$ \\
\hline Levofloxacin & $44.59 \%$ & $40.54 \%$ & $14.86 \%$ \\
\hline Gentamycin & $40.14 \%$ & $40.14 \%$ & $19.71 \%$ \\
\hline Nitrofurantoin & $26.12 \%$ & $38.73 \%$ & $35.13 \%$ \\
\hline Ciprofloxacin & $36.87 \%$ & $27.66 \%$ & $35.46 \%$ \\
\hline Norfloxacin & $13.20 \%$ & $33.96 \%$ & $52.83 \%$ \\
\hline Ampicillin & $\%$ & $45 \%$ & $55 \%$ \\
\hline Nalidixic acid & & $29.88 \%$ & $70.11 \%$ \\
\hline Cefotaxime & $5.28 \%$ & $12.74 \%$ & $81.37 \%$ \\
\hline Amoxicillin & & 12.50 & $87.50 \%$ \\
\hline
\end{tabular}

H.S=high sensitivity, I.S =Intermediate sensitivity, R=Resisant

The least common isolates are Staphlococcus aureus, a gram positive bacteria showed high sensitivity towards norfloxacin $(66.66 \%)$, imipenem $(53.84 \%)$, nitrofurantoin $(44.44 \%)$ and cotrimoxazole (43\%). It showed high resistant towards amoxicillin (75.86\%), nalidixic acid $(65.20 \%)$ vancomycin $(43.87 \%)$. As shown in Table 5.
Table 5: Susceptibility pattern of Staphylococcus aureus.

\begin{tabular}{|c|c|c|c|}
\hline \multicolumn{4}{|c|}{ Staphylococcus aureus } \\
\hline & H.S & I.S & Resistant \\
\hline Norfloxacin & $66.66 \%$ & $\%$ & $33.33 \%$ \\
\hline Imipenem & $53.84 \%$ & $23.07 \%$ & $23.07 \%$ \\
\hline Nitrofurantoin & $44.44 \%$ & $11.11 \%$ & $44.44 \%$ \\
\hline Cotrimoxazole & $43 \%$ & $31.08 \%$ & $25.88 \%$ \\
\hline Levofloxacin & $41.66 \%$ & $58.33 \%$ & $\%$ \\
\hline Ciprofloxacin & $28.57 \%$ & $50 \%$ & $21.42 \%$ \\
\hline Gentamycin & $28.57 \%$ & $28.57 \%$ & $42.85 \%$ \\
\hline Vancomycin & $27.83 \%$ & $29.14 \%$ & $43.87 \%$ \\
\hline Nalidixic acid & $10.48 \%$ & $24.32 \%$ & $65.20 \%$ \\
\hline Amoxicillin & $9.12 \%$ & $15.02 \%$ & $75.86 \%$ \\
\hline
\end{tabular}

H.S =high sensitivity, I.S =Intermediate sensitivity, R =Resisant

\section{DISCUSSION}

Urinary tract infections are common conditions worldwide and the pattern of antimicrobial resistance varies in different regions. The present study describes the relationships between isolated bacterial agents and antibiotic resistance of UTIs. The sex distribution of patients in our study is consistent with those of other reported studies, showing a statistically predominance of females with UTI (57.74\% of the positive cultures). This result is similar to those reported from many other centers. ${ }^{8}$ The elevated incidence of infection among females is related to difference between the male and female genitourinary systems in anatomy and microflora. ${ }^{9}$

Escherichia coli $(37.41 \%)$ is the most common organism causing urinary tract infection in this study followed by Klebsiella species. This is in accordance with earlier study Ranjbar et al, and Amin et al. ${ }^{10,11}$ The highest percentages of resistance of Escherichia coli causing urinary tract infections were found for cotrimoxazole (96.14\%), norfloxacin (88\%), nalidixic acid $(86.76 \%)$, cefotaxime $(69.88 \%)$, whereas the highest percentages of sensitivity were seen for imipenem $(90.8 \%)$, meropenem (85.4\%), amikacin (79.24\%), levofloxacin (77.21\%), gentamycin (62.26\%), ampicillin (59.92\%) and nitrofurantoin $(51.22 \%)$. Despite these finding resistances to cotrimoxazole and floroquinolone is on rise as evident from different study done worldwide. Resistance to SMX-TMP in E. coli could have been due to acquired mutational alterations of the chromosomal SUL and DFR genes. ${ }^{12}$ By a horizontal transfer of antibiotic resistant gene copies and subsequent integration of resistancedetermining gene sequences from the acquired gene into homologous regions of the chromosomal copies. More than 30 resistant variants of trimethoprim resistant DFR genes and three variants of sulfonamide resistant SUL genes have been described so far. ${ }^{12}$ In a study done in Beijing during 1997-1999, approximately $60 \%$ of E. coli were resistant to ciprofloxacin. ${ }^{13}$ Resistance to ciprofloxacin and levofloxacin in E. coli reached $21.6 \%$ and $20.4 \%$, respectively, of isolates tested in 2005 . In the 
North American Urinary Tract Infection Collaboration Alliance surveillance study, $5.5 \%$ and $5.1 \%$ of urinary $E$. coli isolates from outpatients in the United States and Canada were resistant to ciprofloxacin and levofloxacin, respectively. ${ }^{14}$ In Escherichia coli, mutational alterations in the Fluoroquinolones target enzymes, namely, DNA topoisomerase II (DNA gyrase) and topoisomerase IV, are recognized to be the major mechanisms through which resistance develops. ${ }^{15}$

Sensitivity and resistance towards gentamycin and ampicillin are $(62.26 \%, 37.73 \%)$ and $(59.92 \%, 23 \%)$ respectively. The resistance to antibiotics in our region may be due to a higher rate of antibiotic usage, even in the absence of a prescription. Reducing the number of prescriptions of a particular antibiotic can lead to a decrease in resistance rates. Khotaii et al, reported resistance rates of $87.5 \%$ to ampicillin, $67.5 \%$ to trimethoprim-sulfamethoxazole. ${ }^{16}$ A study done in King Fahd Hospital, Saudi Arabia showed that meropenem was $95.8 \%$ sensitive followed by amikacin $(93.7 \%)$ and imipenem (91.71\%) against extended spectrum $B$ lactamase producing E. coli. ${ }^{17}$

It is worth noting that there is considerable reduction in the activity of Trimethoprim-sulfamethoxazole followed by cefotaxime and also nitrofurantoin among the commonly used drugs in treatment of UTI. This was also noted by Sana et al, in a study done in Kuwait and also in the U.S, southern Europe, Israel, and Bangladesh with up to $50 \%$ of $E$. coli strains being resistant to antibiotics used. ${ }^{12,18}$ In our study, $40.65 \%$ of the isolates were resistant to Nitrofurantoin, which is above $10 \%$ what is common in most areas. ${ }^{18}$ This suggests a likelihood of significant increase in resistance to this drug. ${ }^{19,20}$

Athough the percentage of $E$. coli isolated from urine culture is lower in our study due to emergence of increasing trends of other etiological agent as causative organism for UTI, it still supports the previous findings indicating that $E$. coli is the principal etiological agent of UTI, accounting for $46.98 \%$ of the screened cases. ${ }^{21-23}$

The prevalence and antibiotic sensitivity of Klebsiella strains varied among published literatures, as study from Kuwait University, Kuwait, showed that Klebsiella was accounting for $12.2 \%$ of the organism isolated. ${ }^{24}$ In a study done in Aligarh, India, Klebsiella was isolated in $22 \%$ of cultures of 920 patients with UTI. ${ }^{25}$ In our study the Klebsiella was second most common isolated bacteria (25.86\%) and showed a low degree of sensitivity to most antibiotics tested except an $100 \%$ to cefoperazone with sulbactum, $92.0 \%$ sensitivity to imipenem, $69.64 \%$ to gentamycin, $59.79 \%$ to amikacin. Among low sensitive drug, resistance to amoxicillin were $100 \%$, nalidixic acid (72.41\%), norfloxacin $(55.07 \%)$, ciprofloxacin (35\%).

K. pneumoniae produce various enzymes that target specific parts of drugs and deactivate them. The enzymes produced usually target beta lactam type drugs. These enzymes include extended spectrum beta lactamases, metallo-beta-lactamases, oxacillinases, $K$. pneumoniae carbapenemases, and various others. These enzymes are encoded on plasmids which $K$. pneumoniae seems to readily uptake by conjugation process. Extended spectrum beta-lactamases (ESBLs) are so named due to their ability to hydrolyze a wide spectrum of beta-lactam drugs. Their action occurs through the hydrolyzation of the beta-lactam ring in beta-lactam drugs by nucleophilic attack. ${ }^{26}$ Plasmids encoding Temoniera (TEM) and Sulfhydryl variable (SVH) ESBLs are the most common to be found in isolated $\mathrm{K}$. pneumoniae, which are active against cephaloporins. The plasmids that encode the ESBL genes also have been found to carry genes that express resistance for drugs other than beta-lactams, such as aminoglycosides. ${ }^{27}$

Because carbapenems are not degraded by ESBLs, they are used for treatment when ESBL producing $K$. pneumoniae are isolated from patient samples, other mechanism includes biofilm production, efflux pump. Biofilms protects the $K$. pneumoniae from antibiotic treatments. This protection, originally thought to be a result of limited penetration of antibiotic molecules and results of slow growth of pathogens at the center of the biofilm. ${ }^{27}$

P. aeruginosa is the third most common pathogen associated with hospital-acquired catheter associated UTIs. ${ }^{28}$ Virulence of $P$. aeruginosa is multifactorial and has been attributed to cell associated factors like alginate, lipopolysaccharide (LPS), flagellum, virulence factors like protease, elastase, phopholipase, pyocyanin, exotoxin A, exoenzyme S, hemolysins (rhamnolipids) and siderophores, shown to play an important role in pathogenesis of $\mathrm{P}$. aeruginosa induced infections like respiratory tract infections, burn wound infections and keratitis. ${ }^{29,30}$ However, limited reports are available regarding role of these virulence traits in urinary tract infections. Woods et al. showed high production of elastase and protease in strains isolated from urinary tract infections in comparison to isolates from other infections like burn wounds infection, skin wound infection and acute pneumonia and still with above host factor in preventing UTI the present study showed Pseudomonas to be $3^{\text {rd }}$ most common organism causing UTI. ${ }^{31}$ Here, Pseudomonas showed the highest sensitivity to amikacin, then toward gentamicin and absolute resistance to chloramphenicol, nitrofurantoin, cephalexin, coamoxyclav, and tetracycline. In present study Pseudomonas showed high sensitivity towards imipenem $(87 \%)$, ceftriaxone with sulbactum $(75 \%)$.

S. aureus is a relatively infrequent urinary tract isolate in the general population. In the present study Staphylococcus accounted for $3.92 \%$ of UTI which is higher when compared to finding obtained from other study like in a multicenter, community-based study conducted in Great Britain, S. aureus accounted for only $0.5 \%$ of isolates. ${ }^{32}$ A similar laboratory-based study 
conducted in France found that S. aureus accounted for only $1.3 \%$ of isolates from urine specimens submitted from the community. ${ }^{33}$

Prior studies suggest that isolation of S. aureus from the urine is often secondary to staphylococcal bacteremia originating at another site (e.g., in cases of endocarditis). ${ }^{34}$ Isolation of $\mathrm{S}$. aureus from urine samples in the absence of bacteremia is therefore often considered to represent colonization. It is problematic to define the exact role of $\mathrm{S}$. aureus as a cause of symptomatic urinary tract infection, as opposed to colonization, in this population.

Long-term care patients have a high frequency of asymptomatic bacteriuria. ${ }^{35}$ There is evidence to suggest that the majority of febrile episodes in long-term care patients with bacteriuria are not, in fact, due to urinary tract infection. ${ }^{36}$ Furthermore, long-term care patients may have atypical symptoms in response to true infection. ${ }^{37}$ Thus, a significant problem in interpreting prior studies lies in the inherent difficulty in making a definitive diagnosis of urinary tract infection in the longterm care population.

In our study Staphylococcus was found sensitive to norfloxacin $(66.66 \%)$, imipenem $(53.84 \%)$, nitrofurantoin $(44.44 \%)$ whereas resistance towards amoxicillin were (75.86\%), nalidixic acid $(65.20 \%)$, vancomycin $(43.87 \%)$ and gentamycin (42.85\%). A study conducted by Adebola Onanuga et al, reported of higher resistance of Staph. aureus to gentamicin $(73.9 \%)$, (39.1\%) to nitrofurantoin and $(69 \%)$ to vancomycin. ${ }^{38}$ Whereas in our study, the resistance to gentamicin is low $(42.85 \%)$ but the resistance to nitrofurantoin and vancomycin is quite similar. It is similar to the findings of Olayinka et al who reported $57.7 \%$ resistance in vancomycin in hospital associated S. aureus isolates in Zaria, Nigeria. ${ }^{39}$ Getanet Beyene et al reported $100 \%$ of $K$. pneumoniae isolates resistant to amoxicillin and ampicillin which is similar to our study. ${ }^{40}$

\section{CONCLUSION}

Organisms isolated from urine culture in decreasing order were E. coli, Klebsiella, Pseudomonas and S. aureus, E. coli shows highly sensitive towards imipenem, meropenem, amikacin and nitrofurantoin. It showed high resistance towards commonly prescribed drug for UTI i.e. found for cotrimoxazole, norfloxacin, nalidixic acid, cefotaxime. Klebsiella the second most common isolates which showed high sensitivity to cefoperazone with sulbactum, aminoglycosides like gentamicin and amikacin. It showed high resistance to amoxicillin, nalidixic acid and norfloxacin. The third most common isolates were Pseudomonas which showed high sensitivity to imipenem, ceftriaxone with sulbactum and amikacin with resistance to commonly prescribed drug like amoxicillin, cefotaxime and nalidixic acid.
Staphylococcus though a rare cause of UTI is showing high sensitivity towards norfloxacin, imipenem, nitrofurantoin and high resistant towards amoxicillin, nalidixic acid and vancomycin.

High level of antimicrobial resistance percentage in this study indicates the appearance of resistance towards commonly used antimicrobials agent such as first line drugs (amoxicillin, ampicillin and cefotaxime), this may be due to the widespread and prolonged use of these drugs for empirical therapy that may have an impact on the antibiotic treatment of UTI patients. The use of ampicillin, amoxicillin and cefotaxime as first line empirically therapy in UTI is causing lot of resistance so it should not be used empirically. The results of this study may not be representative of the general population; but UTIs are often treated empirically, and susceptibility tests are often carried out only when the patient has failed one or more courses of antibiotics. These data may be used to determine trends in antimicrobial susceptibilities, to formulate local antibiotic policies, to compare local with national data and overall to assist clinicians in the rational choice of antibiotic therapy to prevent misuse or overuse of antibiotics. As there is derth of new molecules of antibiotics for the management of UTI we need to be concerned for this in future. A unified antibiotic protocol is therefore necessary to restrict use of antibiotics injudiciously in order to prevent resistance and reduce the complication of UTI arising from use of resistant drugs.

In this study only limited numbers of drugs were studied for sensitivity pattern and culture was done for common pathogenic organisms and mechanism of resistance were elaborated in brief of only few drugs. There is need for wide range and periodical study to know the changing sensitivity pattern of microorganisms. This study may be helpful in deciding empirical therapy of an infection considering other related factors before the actual culture and sensitivity report of a microorganism comes.

Funding: No funding sources

Conflict of interest: None declared

Ethical approval: The study was approved by the Institutional Ethics Committee

\section{REFERENCES}

1. Ronald AR. The natural history of urinary infection in adults. Med Clin North Am. 1991;75:299-312.

2. Bano S, Tunio SA, Menom AA, Detho H, Bano R, Kumari K. Evaluation of antibiotic susceptibility patterns of uropathogens circulating in Hydrabad, Pakisthan. Khyber Med Univ J. 2014;6(3):110-5.

3. Biswas R, Rabbani R, Ahmed HS, Abdus M, Sarkar $\mathrm{S}$, Zafrin N, et al. Antibiotic sensitivity pattern of urinary tract infection at a tertiary care hospital. Bangladesh Crit Care J. 2014;2(1):21-4.

4. Saint S. Clinical and economic consequences of nosocomial catheter related bacteriuria. Am J Infect Control. 2000;28:68-75. 
5. Das RN, Chandrashekhar TS, Joshi HS, Gurung M, Shrestha N, Shivananda. P G Department. Frequency and susceptibility profile of pathogens causing urinary tract infections at a tertiary care hospital in western Nepal. Singapore Medica Journal. 2006;47(4):281.

6. Nicole W, Jon DM. Deciphering Dysuria. Emerg Med. 2008;40(9):29.

7. Jenson BH, Baltimore RS. Infectious Diseases. Nelson Essentials of Pediatrics 5th edition. Elsevier Inc. 2006;522.

8. Abu SQ. Occurrence and antibiotic sensitivity of Enterobacteriaceae isolated from a group of Jordanian patients with community acquired urinary tract infections. Cytobios. 2000;101:15-21.

9. Strom BL, Collins M, West SL, Kreisberg J, Weller S. Sexual Activity, Contraceptive Use, and Other Risk Factors for Symptomatic and Asymptomatic BacteriuriaA Case-Control Study. Annals of internal medicine. 1987 Dec 1;107(6):816-23

10. Ranjbar R, Haghi AM, Jafari NJ, Abedini M. The prevalence and antimicrobial Susceptibility of bacterial uropathogens isolated from paediatric patients. Iranian J Pub Health. 2009;38(2):134-8.

11. Amin M, Mehdinejad M, Pourdangchi Z. Study of bacteria isolated from urinary tract Infections and determination of their susceptibility to antibiotics. Jundishapur J Microbiol. 2011;2(3):118-23.

12. Sköld, O. Sulfonamides and trimethoprim. Exp. Rev. Anti-Infect. Ther. 2010;8(1):1-6. Available at https://www.ncbi.nlm.nih.gov/labs/articles/20014895 I

13. Wang H, Dzink-Fox JL, Chen M, Levy SB. Genetic characterization of highly fluoroquinolone-resistant clinical Escherichia coli strains from China: Role of acrr mutations. Antimicrobial Agents and Chemotherapy. 2001;45(5):1515-21.

14. Naber KG, Llorens L, Kaniga K, Kotey P, Hedrich $\mathrm{D}$, Redman R. Intravenous doripenem at 500 milligrams versus levofloxacin at 250 milligrams, with an option to switch to oral therapy, for treatment of complicated lower urinary tract infection and pyelonephritis. Antimicrobial Agents and Chemotherapy. 2009;53(9):3782-92.

15. Karczmarczyk M, Martins M, Quinn T, Leonard N, Fanning S. Mechanisms of Fluoroquinolone Resistance in Escherichia coli Isolates from FoodProducing Animals. Applied and Environmental Microbiology. 2011;77(20):7113-20.

16. Khotaii Q, Mamishi S, Saligeh RN. Antibiotic resistance of germs isolated from urinary tract infections. Iran J Pediatr. 2002;12:28-32.

17. Al-Zahran AJ, Akhtar N. Susceptibility patterns of extended spectrum beta-lactamase (ESBL)-producing Escherichia coli and Klebsiella pneumoniae isolated in a teaching hospital, Pakistan Journal of Medical Research. 2005;44:64-7.

18. Al-Ali SM, Al-Hamdan AS, Al-Jark FA, Al-Faraj JMK, Mussalem SSA. Antimicrobial resistance pattern in urinary tract pathogens and its impact on empirical therapy in general practice, Kuwait Medical Journal. 2005;37(1):22-7.

19. Manges AR, Johnson JR, Foxman B, O'Bryan TT, Fullerton KE, Riley LW. Widespread distribution of urinary tract infections caused by a multi drug resistant Escherichia coli clonal group. New England Journal Medicine. 2001;345:07-1013.

20. Warren JW, Abrutyn E, Hebel JR, Johnson JR, Schffer AJ, Stamm WE. Guidelines for antimicrobial treatment of uncomplicated acute bacterial cyctitis and acute pyelonephritis in women. J. Clinical infection Disease. 1999;29:745-58.

21. Jha N, Bapat SK. A study of sensitivity and resistance of pathogenic microorganism causing UTI in Kathmandu valley. Kathmandu Univ Med J. 2005;3:123-9.

22. Ronald A. The quinolones and renal infection Drugs, 1999;58:96-8. Available at: https://www.researchgate.net/publication/12746649 The_Quinolones_and_Renal_Infection.

23. Ronald A. The etiology of urinary tract infection: traditional and emerging pathogens. Am J Med. 2002;113:14-9.

24. Dimitrov TS, Udo EE, Emara M, Awni F, Passadilla R. Etiology and antibiotic susceptibility patterns of community-acquired urinary tract infections in a Kuwait hospital. Med Princ Pract. 2004;13:334-3.

25. Akram M, Shahid M, Khan Au. Etiology and antibiotic resistance patterns Of Communityacquired urinary tract infections in JNMC Hospital Aligarh, India. Ann Clin Microbiol Antimicrob. 2007;23:6-4.

26. Papp-Wallace KM, Endimiani A, Taracila MA, Bonomo RA. Carbapenems: Past, Present, and Future. Antimicrobial Agents and Chemotherapy. 2011;55(11):4943-60.

27. Vuotto C, Longo, F, Balice MP, Donelli G, Varaldo, PE. Antibiotic Resistance Related to Biofilm Formation in Klebsiella pneumoniae. Pathogens. 2014;3(3):743-58.

28. Jarvis WR, Martone WJ. Predominant pathogens in hospital infections. J Antimicrob Chemother. 1992;29:19-24.

29. Matheson NR, Potempa J, Travis J. Interaction of a novel form of Pseudomonas aeruginosa alkaline protease (aeruginolysin) with interleukin-6 and interleukin-8. Biol Chem. 2006;387:911-5.

30. Lysczak JB, Cannon CL, Pier GB. Establishment of Pseudomonas aeruginosa infection: lessons from a versatile opportunist. Microbes Infect. 2000;2:105160.

31. Woods DE, Schaffer MS, Rabin HR, Campbell GD, Sokol PA. Phenotypic comparison of Pseudomonas aeruginosa strains isolated from a variety of clinical sites. J Clin Microbiol. 1986;24:260-4.

32. Barrett SP, Savage MA, Rebec MP, Guyot A, Andrews N, Shrimpton SB. Antibiotic Sensitivity of bacteria associated with community acquired urinary tract infection in Britain. $J$ Antimicrob Chemother.1999;44:359-6. 
33. Goldstein FW. Antibiotic susceptibility of bacterial strains isolated from patients with Communityacquired urinary tract infections in France. Eur J Clin Microbiol Infect Dis. 2000;19:112-7.

34. Musher DM, Mckenzie SO. Infections due to Staphylococcus aureus. Medicine. 1977;56:383-409.

35. Breitenbucher RB. Bacterial changes in urinary samples of patients with long-term indwelling catheters. Arch Intern Med. 1984;144:1585-8.

36. Nicolle LE, Muir P, Harding GKM, Norris $M$. Localization of urinary tract infection in elderly, institutionalized women with asymptomatic bacteriuria. J Infect Dis. 1988;157:65-70.

37. Jones JW, Carter A, Ewings P, O'Boyle PJ. An MRSA outbreak in a urolgy ward and its association with Nd: YAG coagulation laser treatment of the prostate. J Hosp Infect. 1999;41:3944.

38. Onanuga A. Antimicrobial resistance of S. aureus strains from patients with urinary tract infections in
Yenagua, Nigeria. J. Of Phar and Bioallied Sci. 2012,Jul-Sept;4(3):226-30.

39. Olayinka BO, Olayinka AT, Onaolapo JA, Olurinola PF. Pattern of resistance to vancomycin and other antimicrobial agents in staphylococcal isolates in a university teaching hospital. Afr. Clin. Exper. Microbiol. 2005;6:46-52.

40. Beyene G, Tsegaye W. Bacterial uropathogens in urinary tract infection and antibiotic susceptibility pattern in JIMMA university specialized hospital, Southwest Ethiopia. Ethiopian Journal of Health Sciences. 2011;21(2):141-6.

Cite this article as: Kumar A, Kumar R, Gari M, Keshri UP, Mahato SK, Kumari R. Antimicrobial susceptibility pattern of urine culture isolates in a tertiary care hospital of Jharkhand, India. Int J Basic Clin Pharmacol 2017;6:1733-9. 